\title{
Sensor Authentication in Dynamic Wireless Sensor Network Environments
}

\author{
Kyusuk Han \\ KAIST, Korea
}

\author{
Taeshik Shon \\ Ajou University
}

\begin{abstract}
While applications of Wireless Sensor Network are diversified, several new issues such as mobility of sensor node are raised and bring security issues such as re-authentication and tracing the node movement. In the dynamic sensor network, mobile sensor nodes will continuously move around and frequently reconnect to other sensor nodes. While many security protocols to such networks occur significantly large overheads because their design only considered the static networks. There are several studies on such dynamic environments. In this paper, we show our design for the efficient node authentication and key exchange that reduces the overhead in node re-authentication and also provides untraceablity of mobile nodes. We introduce protocols that are symmetric key crypto system based and public key crypto system based. We also introduce the application scenario of the protocol that is integrated to other networks.
\end{abstract}

\section{Introduction}

Wireless Sensor Network (WSN) is the network that consists of lightweight devices with short-ranged wireless communication and battery-powered. The devices have the sensor that gathers the environmental information and etc. After sensing this information, the devices send the information to the networks. We define such devices as sensor node, and the core parts of the network as sinks and the base station.

Rapid development of WSNs brought themselves to be deployed to various areas such as RF4CE [19]. However, many security studies on WSNs are rather inefficient to be deployed to such environments.

While there are many trials for providing efficient security functions for WSN such as $[4,5$, $13,14]$, as one of the fundamental security issues, there are various researches on key management in WSN such as key-pre distribution, pairwise key agreement, group key based key agreement, and hierarchical key management schemes. There were also trials of PKI deployment for WSN [17]. In order to reduce the communication overhead from the key establishment, Huang et al. [10] proposed public key infrastructure (PKI) based model applying Elliptic
Curve Cryptography [15]. However, applying PKI requires larger computational power, although it enables the simplified key agreement procedure.

Since it is obvious that the wireless sensor network will be widely deployed by combining network of static sensor network and mobile entities such as [2, 6], handling a large overhead from frequent node re-authentication requests due to the continuous node movements and the threats of tracing the node movement will be important security issues.

In order to solve such problems, we proposed efficient authentication models that significantly reduce overhead for re-authenticating sensor nodes $[7,8]$.

In this paper, we show our model that provide high efficiency in such environments and shows several protocols that we previous introduced in [7, $8]$.

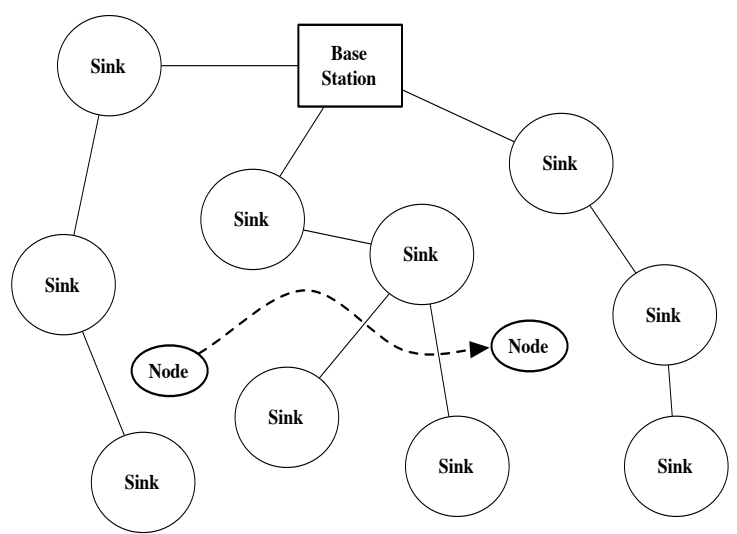

Figure 1 A dynamic mobile node continuously moves in the sensor networks that static sinks established. The unbroken line denotes the static connection between sinks and the base station. The dotted line denotes the movement of the mobile node.

\section{Our Sensor Authentication Model}

\subsection{Dynamic WSN Environment}

In this section, we claim the security issues on the node mobility in WSN and problems of previous authentication and key agreement models. In paper 
[kyusuk1, kyusuk2, kyusuk3], we defined a sensor network model with moving nodes as in Figure 1. We also defined a static sensor node as Sink, a mobile node as Node, and the base station that is the core network. The node has linear movements in the network. The base station and sinks are static as same as Ibriq and Mahgoub's model [11]. Sinks act as the gateway that link nodes to the base station, and the base station is a kind of headquarter that manages entire networks. When a node initially joins the network, the node connects to a sink in the network and is authenticated by the sink with help of the base station. After that the node moves and reconnects to other sink. In the model, the sink that reauthenticates the node is the neighbor sink of the sink that previously authenticated the node. The reauthentication processes frequently happen due to the node continuously moves in the network.

In practical scenarios, re-authentication happens when a node lost connection to the sink or moved and connected to other sink. For the former case, the node can be easily re-authenticated to the same sink when the connection becomes available again. For the latter case, the node request the re-authentication to other sink that is the near to the previously attached sink.

For such environments several security Issues raise as followings.

2.1.1. Frequent Re-authentication. Since the sensor have low powered battery and low-end processor with short-range wireless communication, the reducing communication and computational overheads is important to increasing the lifetime of the sensor. However, the mobile sensor node may occur the large overhead for the security computation due to the frequent requests of node reauthentication. When a node connects to a sink, the sink has to authenticate the node. When the node connects to other sink after the movement, the new sink has to authenticate the node again. If the node has continuous movement, the authentication process will also occur repeatedly. It is obvious that the frequent re-authentication processes are the significant factors that drain the resources in batterybased sensor nodes.

However, the current authentication and key distribution protocols are insufficient to be applied in such environment with lack of consideration of the node mobility. Using the current protocols such as $[11,1]$, the communication overhead for reauthentication is as same as previous authentication.

Such overhead will be the problem in the environment that the frequent movements of the large number of nodes are happened. Thus, the less computational and communication overheads in reauthentication are very urgent requirement for the node mobility support in the WSN.
2.1.2. Tracing Node Movements. Considering the mobility of sensor nodes, the tracking of the node movement is one of possible attacks. When the mobile nodes are deployed in battlefields, the tracking by enemies is significant threats for the networks. Thus, the authentication and key agreement protocols should not reveal the node movement.

\subsection{Security Requirements}

We defined the security requirements as follows. We assume that when the node $N$ communicates with a sink $S_{2}$ after disconnection to the sink $S_{1}, S_{1}$ cannot receive any message between $N$ and $S_{2} . S_{2}$ is one of neighbor sinks of $S_{l}$.

- Re-authentication An authenticated node $N$ and $S_{2}$ should be able to identify each other with less communication and computational overhead than initial authentication.

- Untraceablity In re-authentication of $N, S_{2}$ only knows that $N$ was previously connected to $S_{l}$, and never knows the direction of $N$.

- Confidentiality When $N$ and $S_{1}$ are operating initial authentication, nobody can know the communication packet between $N$ and $S_{l}$, between $S_{I}$ and $B S$. For re-authentication between $N$ and $S_{2}$, nobody except $S_{I}$ can know the communication information, while $S_{l}$ out of communication range.

- Message Integrity Any malicious adversaries should not be able to forge the communication packet.

- Key Freshness $N$ and $S$ should be able to verify that the key is generated during the current session.

- Node/Sink Resiliency Even $N, S_{1}$ or $S_{2}$ are compromised by a malicious adversary, they should not be able to affect to the entire network.

'Confidentiality', 'message Integrity', and 'key freshness' are important requirements against the replay attack or man-in-the-middle attack. 'Node/Sink resiliency' is practical threat that the sensor nodes are generally deployed in the environment out of administration.

\section{Efficient Sensor Authentication Protocol for Dynamic Environments}

\subsection{Overview of Our Model}

We briefly describe our proposed protocol. Assume that there are a base station $B S$, a sink $S_{l}$, a neighbor sink $S_{2}$, and a mobile node $N$ in the 
network. $S_{l}$ periodically broadcasts HELLO in Phase 0 . When $S_{2}$ receives HELLO, $S_{2}$ initiates the neighbor relationship if $S_{I}$ is a newly discovered sink. After the pairwise key between $S_{I}$ and $S_{2}$ has been exchanged in Phase $1, S_{1}$ and $S_{2}$ exchange the authentication key that is used to verify the authenticated user in Phase 2. Phase 1 and Phase 2 are bootstrapping phase for the node reauthentication.

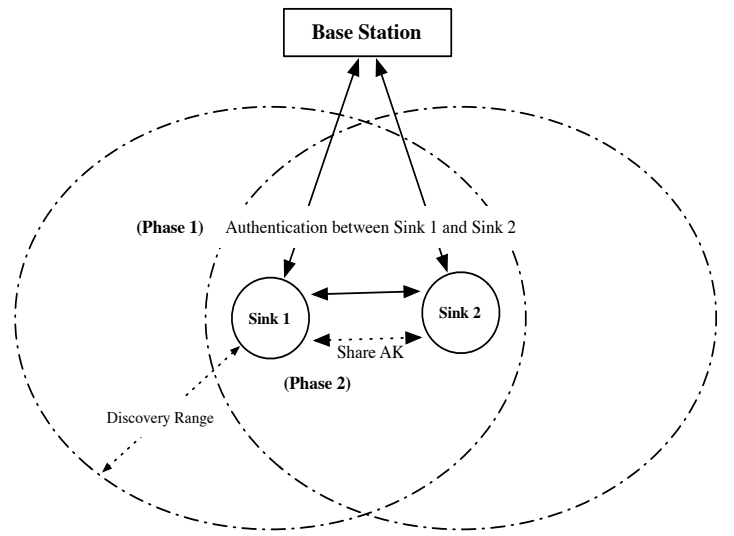

Figure 2 Sink $1\left(S_{1}\right)$ mutually authenticate Sink 2 (Phase 1), and share the authentication key (Phase 2).

When $N$ that is not authenticated by any sink joins the network, $N$ receives HELLO of $S_{1}$ and initiates the initial node authentication with $S_{l}$ in Phase 3. Later, $N$ moves and reconnects to $S_{2}$. Then $N$ initiates the node re-authentication in Phase 4 . Figure 2, 3 shows each phase of our model.

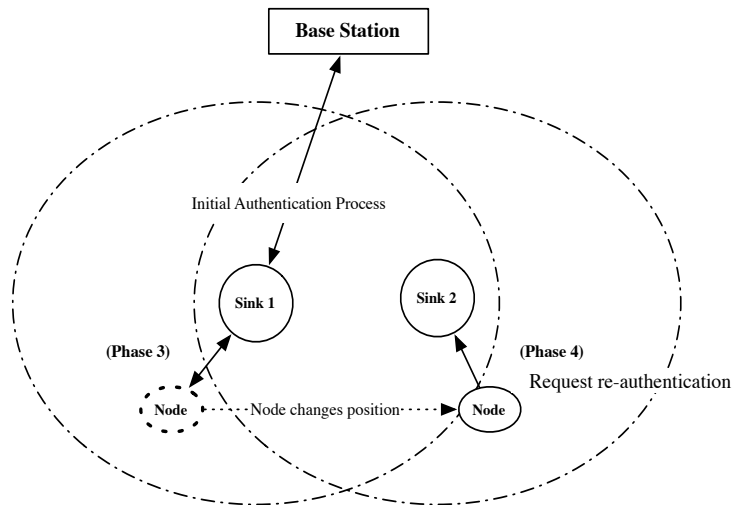

Figure 3 Node is initially authenticated by Sink 1 (Phase 3), and requests re-authentication to Sink 2.

Thus our model consists of following five phases.

- Phase 0 The common neighbor discovery

- Phase 1 Setting up neighbor sink relationship

- Phase 2 Neighbor group authentication key share

- Phase 3 Initial node authentication

- Phase 4 Node re-authentication
3.1.1. Authentication Ticket. We define 'Authentication Ticket' that is used for the node reauthentication. When a node requests authentication to a sink, the sink generates the authentication ticket and sends it to the node. The authentication ticket is verified by the authentication key shared to neighbor sinks. Using the authentication ticket, the node movement is untraceable. Verification of the authentication ticket is available to neighbor sinks of the sink that issued the ticket. We adopt the idea of 'cluster key' in [LEAP+] that shared to neighbor sinks. The main difference is that the role of cluster key is very limited to verifying the authentication ticket. Thus, we rename the key as 'authentication key' due to the limited use in the protocol.

3.1.2. Neighbor Sink List. Assume that static sink nodes are distributed as shown in Figure 4 (a). In this case, the node authenticated by $S_{3}$ can be reauthenticated from the neighboring sinks $S_{1}$ or $S_{4}$, wherever it moves. However, the sinks may not be well distributed in the real environments.

In Figure 4 (b), a node that is authenticated by $S_{l}$ cannot directly be re-authenticated by $S_{5}$, since $S_{5}$ is not a neighbor sink of $S_{l}$. However, we also see that both $S_{1}$ and $S_{5}$ have the common neighbor sink $S_{2}$ that may link $S_{1}$ and $S_{5}$ for the re-authentication of $N$.

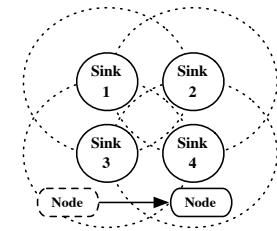

(a) Ideal Environments

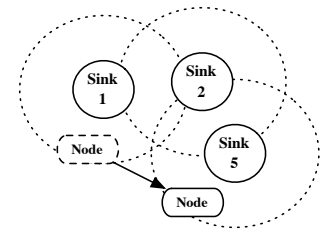

(b) Real Environments
Figure 4: Static sensor node distribution in (a) Ideal environments (b) Real Environments

Thus, we defined the NSL that stores the neighboring sink's information such as their ID, shared secret key, and public key. We assume that each sink has its own NSL. Let the NSL stored in $S_{l}$ be $N S L_{S_{1}}=t \| \operatorname{sign}_{S_{1}}(h(t))$, where $t=S_{1}\left\|S_{2}\right\| \ldots . \| S_{k}$ and $\operatorname{sign}_{S_{1}}$ is the signature of $S_{l}$ as in Table 1. Figure 8 shows that $S_{1}$ and $S_{5}$ have a common sink $S_{2}$ that can link the two sinks. The signature scheme can be flexibly chosen. When TinyECC [15] is used, the Elliptic Curve Digital Signature Algorithm (ECDSA) can be properly applied. Whenever a new neighboring sink is found, the sink updates its own NSL.

\subsection{Symmetric Key based Protocol}

3.2.1. Pre-Phase 0: Neighbor Discovery. A sink $S_{I}$ periodically generates a random nonce $R_{l} . S_{l}$ also generates

$$
u_{0}=E_{K_{S_{1}}}\left\{R_{0} \| T S_{0}\right\}
$$
and 
$v_{0}=M A C_{K_{S_{1}}}\left(S_{1} \|\right.$ HELLO $\left.\| u_{0}\right)$, where $T S_{0}$ is time stamp. $u_{0}$ and $v_{0}$ are included in the HELLO message. Then $S_{l}$ broadcasts $u_{0}$ and $v_{0}$ as follows:

$$
S_{1} \rightarrow \text { Broadcast }: S_{1} \| \text { HELLO }\left\|u_{0}\right\| v_{0}
$$

Phase 0 is the periodical common procedure. When a sink receives HELLO, the sink initiates Phase 1 or Phase 2. When a node receives HELLO, the node initiates Phase 3 or Phase 4.

3.2.2. Pre-Phase 1: Setting up Neighbor Sink Relationship. Assume another sink $S_{2}$ receives HELLO message. $S_{2}$ checks the sender of HELLO whether $S_{1}$ is known or not. If $S_{2}$ already knows $S_{1}$, $S_{2}$ discards the message. Otherwise, $S_{2}$ requests the setting up the neighbor relationship as follows:

1. $S_{2}$ randomly selects $R_{l}$ and generates $u_{1}=E_{K_{S_{2}}}\left\{R_{1} \| u_{0}\right\}, v_{1}=M A C_{I K_{S_{2}}}\left(S_{2}\|\mathrm{BS}\| S_{1}\left\|u_{1}\right\| v_{0}\right)$.

$$
S_{2} \rightarrow \mathrm{BS}: S_{2}\|\mathrm{BS}\| S_{1}\left\|u_{1}\right\| v_{1} \| v_{0}
$$

2. After verifying $v_{l}$, BS decrypts $u_{l}$ and retrieves $R_{l}$ and $v_{0}$. Then, BS verifies $v_{0}$ and decrypts $v_{0}$. Finally, BS retrieves $R_{0}$ and $T S_{0}$. BS generates and sends $u_{4}$, $v_{4}, \quad$ and $v_{3}$ to $S_{2} \quad$ where, $u_{3}=E_{K_{S_{1}}}\left\{R_{1}\right\}$, $v_{3}=M A C_{I K_{S 1}}\left(\mathrm{BS}\left\|S_{1}\right\| u_{3}\right), \quad u_{4}=E_{K_{2}}\left\{R_{1} \| u_{3}\right\} \quad$ and $v_{4}=M A C_{I K_{2}}\left(\mathrm{BS}\left\|S_{2}\right\| R_{1}\left\|u_{4}\right\| v_{3}\right)$.

$$
B S \rightarrow S_{2}: B S\left\|S_{2}\right\| S_{1}\left\|u_{4}\right\| v_{4} \| v_{3}
$$

3. After verifying $v_{4}, S_{2}$ decrypts $u_{4}$, and retrieves $R_{1}$ and $u_{3} . \quad S_{2}$ generates $K_{S_{1} S_{2}}=K D F\left(0\left\|R_{0}\right\| R_{1}\right) \quad$ and $I K_{S_{1} S_{2}}=K D F\left(1\left\|R_{0}\right\| R_{1}\right)$ with $R_{0}$ and $R_{l} . \quad K_{S_{1} S_{2}}$ is encryption key and $I K_{S_{1} S_{2}}$ is integrity key between $S_{1} \quad$ and $\quad S_{2}$. Then $S_{2}$ generates $v_{5}=M A C_{I K_{S_{1} S_{2}}}\left(S_{2}\left\|S_{1}\right\| R_{0} \| R_{1}\right)$ and sends $u_{3}, v_{3}$, and $v_{5}$ to $S_{l}$.

$$
S_{2} \rightarrow S_{1}: S_{2}\left\|S_{1}\right\| u_{3}\left\|v_{3}\right\| v_{5}
$$

4. After verifying $v_{3}, S_{l}$ decrypts $u_{3}$ and retrieves $R_{l}$. $S_{l}$ also generates $K_{S_{1} S_{2}}$ and $I K_{S_{1} S_{2}}$. Then $S_{l}$ verifies $v_{5}$. $S_{1}$ generates $v_{6}=M A C_{I K_{S_{1} S_{2}}}\left(S_{1}\left\|S_{2}\right\| A C K\left\|R_{0}\right\| R_{1}\right)$ and sends $v_{6}$ with ACK to $S_{2}$.

$$
S_{1} \rightarrow S_{2}: S_{1}\left\|S_{2}\right\| A C K \| v_{6}
$$

5. $S_{2}$ verifies $v_{6}$ and shares pairwise keys $K_{S_{1} S_{2}}$ and $I K_{S_{1} S_{2}}$.

3.2.3. Phase 2: Neighbor Group Authentication Key Share. Phase 2 can be operated solely or after Phase 1 is completed. In Phase 2, $S_{1}$ initiates following procedures.
1. $S_{l}$ randomly selects nonce $A S E E D_{S_{1}}$ and $R_{l}$. Then $S_{1} \quad$ generates $u_{1}=E_{K_{S_{1} S_{2}}}\left\{A S E E D_{S_{1}} \| R_{1}\right\} \quad$ and $v_{1}=M A C_{I K_{S_{1} S_{2}}}\left(S_{1}\left\|S_{2}\right\| u_{1}\right)$.

$$
S_{1} \rightarrow S_{2}: S_{1}\left\|S_{2}\right\| u_{1} \| v_{1}
$$

2. After verifying $v_{l}, S_{2}$ decrypts $u_{1}$, and retrieves ${ }^{A S E E D} S_{S_{1}}$ and $R_{1}$. Then $S_{2}$ generates $A K_{S_{1}}=K D F\left(0 \| A S E E D_{S_{1}}\right)$ and $A I K_{S_{1}}=K D F\left(1 \| A S E E D_{S_{1}}\right)$. $S_{2}$ also generates $v_{2}=M A C_{A I K_{S_{1}}}\left(S_{2}\left\|S_{1}\right\| \mathrm{ACK} \| R_{1}\right)$ using $A I K_{S_{1}}$.

3. $S_{1}$ verifies $v_{2}$.

$$
S_{2} \rightarrow S_{1}: S_{2}\left\|S_{1}\right\| \mathrm{ACK} \| v_{2}
$$

After the Phase 2 is completed, sinks share their neighbor sink's authentication keys.

3.2.4. Phase 3: Initial Node Authentication. When $N$ receives HELLO that $S_{l}$ broadcasts in Phase 0 and is not yet authenticated by any sink, $N$ proceeds followings.

1. Node $N$ randomly selects $R_{I}$ and generates $u_{1}=E_{K_{N}}\left\{R_{1}\left\|u_{0}\right\| v_{0}\right\}$ and $v_{1}=M A C_{I K_{N}}\left(N_{1}\left\|S_{1}\right\| u_{1}\right)$.

$$
N \rightarrow S_{1}: N\left\|S_{1}\right\| u_{1} \| v_{1}
$$

2. $S_{1}$ generates $v_{2}=M A C_{I K_{S_{1}}}\left(S_{1}\|\mathrm{BS}\| N\left\|u_{1}\right\| v_{1}\right)$.

$$
S_{1} \rightarrow \mathrm{BS}: S_{1}\|\mathrm{BS}\| N\left\|u_{1}\right\| v_{1} \| v_{2}
$$

3. After verifying $v_{2}$ and $v_{l}$, BS decrypts $u_{l}$, and retrieves $R_{0}, u_{0}$ and $v_{0}$. After verifying $v_{0}$, BS decrypts $u_{0}$, and retrieves $R_{0}$ and TS. BS checks the validity of TS and generates $u_{3}=E_{K_{N}}\left\{R_{0}\right\}$, $v_{3}=M A C_{I K_{N}}\left(B S\|N\| S_{1} \| u_{3}\right), \quad u_{4}=E_{K_{S_{1}}}\left\{R_{1}\left\|u_{3}\right\| v_{3}\right\} \quad$ and $v_{4}=M A C_{I K_{S_{1}}}\left(B S\left\|S_{1}\right\| N\left\|R_{0}\right\| u_{4}\right)$.

$$
B S \rightarrow S_{1}: B S\left\|S_{1}\right\| N\left\|u_{4}\right\| v_{4}
$$

4. After verifying $v_{4}, S_{l}$ decrypts $u_{4}$, and retrieves $R_{l}$, $u_{3}$ and $v_{3}$. Then $S_{1}$ generates $N K_{N}=K D F\left(R_{0} \| R_{1}\right) . S_{I}$ generates $\quad t=E_{A K_{S_{1}}}\left\{T S\left\|R_{1}\right\| N K_{N}\right\} \quad$ and


$u_{5}=E_{N K_{N}}\{T S\|t\| w\}$ and $v_{5}=M A C_{N I K_{N}}\left(S_{1}\|N\| R_{0} \| u_{5}\right)$.

$$
S_{1} \rightarrow N: S_{1}\|N\| u_{3}\left\|v_{3}\right\| u_{5} \| v_{5}
$$

5. After verifying $v_{3}, N$ decrypts $u_{3}$ and retrieves $R_{0}$. Then $N$ also generates $N K_{N}$ and verifies $v_{5} . N$ decrypts $u_{5}$ and retrieves $T S, t$ and $w . N$ generates $v_{6}=M A C_{N K_{N}}\left(N\left\|S_{1}\right\| A C K\left\|R_{0}\right\| R_{1}\right)$.

$$
N \rightarrow S_{1}: N\left\|S_{1}\right\| \mathrm{ACK} \| v_{6}
$$

6. $S_{l}$ verifies $v_{6}$ 
3.2.5. Phase 4: Node Re-authentication. When $N$ receives HELLO that $S_{2}$ broadcasts in Phase 0 and is previously authenticated by a sink, $N$ proceeds followings.

1. $N$ generates $v_{1}=M A C_{N I K_{N}}\left(N\left\|S_{2}\right\| t\|w\| v_{0}\right)$.

$$
N \rightarrow S_{2}: N\left\|S_{2}\right\| t\|w\| v_{1}
$$

2. $S_{2}$ verifies $w$ and decrypts $t . S_{2}$ retrieves $R_{l}, N K_{N}$ and $T S$. Using $N K_{N}, S_{2}$ verifies $v_{l}$. Then $S_{2}$ generates $N K^{\prime}=K D F\left(R_{1} \| R_{0}\right)$, also generates $t^{\prime}=E_{A K_{S_{2}}}\left\{R_{1} \| N K_{N}^{\prime}\right\}$ and $w^{\prime}=M_{A A C_{A I K_{S_{2}}}}\left(N \| t^{\prime}\right) . S_{2}$ generates $v_{2}=h\left(N K_{N}^{\prime} \| R_{0}\right)$ and $u_{3}=E_{N K_{N}}\left\{R_{0}\left\|v_{2}\right\| t^{\prime} \| w^{\prime}\right\}, v_{3}=M A C_{N I K_{N}}\left(S_{2}\|N\| u_{3}\right)$.

$$
S_{2} \rightarrow N: S_{2}\|N\| u_{3} \| v_{3}
$$

3. After verifying $v_{3}, N$ decrypts $u_{3}$ and retrieves $R_{0}$, $v_{2}, t^{\prime}$ and $w^{\prime}$. Then $N$ generates $N K_{N}^{\prime}$ and verifies $v_{2}$. $N$ generates $v_{4}=M A C_{N I K_{N}^{\prime}}\left(N\left\|S_{2}\right\| \mathrm{ACK}\left\|R_{0}\right\| R_{1}\right)$.

$$
N \rightarrow S_{2}: N\left\|S_{2}\right\| \mathrm{ACK} \| v_{3}
$$

4. After verifying $v_{4}, S_{2}$ authenticates $N$.

\subsection{PKI-deployed Protocol}

PKI-deployed protocol has a slightly different process from symmetric key based protocol. It uses Neighbor Sink List, introduced in section 3.1.2.

3.3.1. Pre-Phase 0: Neighbor Discovery. A sink $S_{l}$ generates $v_{0}=\operatorname{sign}_{s k_{1}}(h(H E L L O \| T S))$, where HELLO is a generic "HELLO" message and TS is the time stamp. $S_{l}$ broadcasts $v_{0}$ with HELLO,TS, $p k_{l}$, and cert $_{S_{1}}$. Because only $p k_{1}$ and cert $_{S_{1}}$ are required for pre-phase1, any nodes that already know $p k_{1}$ and cert $_{S_{1}}$ will ignore this phase when they receive the HELLO message.

3.3.2. Pre-Phase 1: Neighbor Sink List Set Up. When a sink $S_{2}$ receives the "HELLO" message of $S_{1}$ that has no previous relationship with $S_{2}, S_{2}$ operates as follows:

a. After verifying cert $t_{S_{1}}$ using $p k_{B S}, S_{2}$ verifies $v_{0}$ using $p k_{l}$. Then, $S_{2}$ randomly selects $R_{l}$, generates $u_{1}=e n c_{p k_{1}}\left\{S_{2}\left\|S_{1}\right\| R_{1} \| h\left(R_{1}\right)\right\}$, and returns $u_{1}$ to $S_{1}$ as follows.

$$
S_{2} \rightarrow S_{1}: S_{2}\left\|S_{1}\right\| u_{1}
$$

b. $S_{1}$ also verifies $p k_{2}$ and retrieves $R_{1}$ after decryption of $u_{1}$. $S_{1}$ then randomly selects $R_{2}$ and generates $K_{S_{1} S_{2}}=K D F\left(R_{1} \| R_{2}\right) . K D F$ is a key derivation function such as the hash function. $S_{I}$ generates $u_{2}=e n c_{p k_{2}}\left\{S_{1}\left\|S_{2}\right\| R_{2} \| h\left(K_{S_{1} S_{2}} \| R_{2}\right)\right\}$ and sends $u_{2}$ to $S_{2}$ as follows.

$$
S_{1} \rightarrow S_{2}: S_{1}\left\|S_{2}\right\| u_{2}
$$

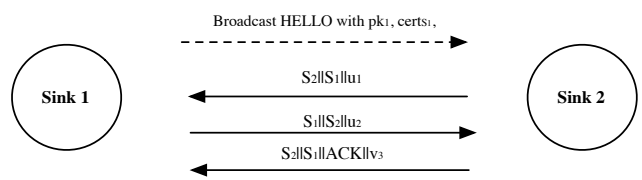

Figure 5: Pre-phase 1: Neighbor Sink List Set Up

c. $S_{2}$ decrypts $u_{2}$ and retrieves $R_{2} . S_{2}$ also generates $K_{S_{1} S_{2}}$ and checks $h\left(K_{S_{1} S_{2}} \| R_{2}\right)$ for freshness check. $S_{2}$ generates $v_{3}=M A C_{K_{S_{1} S_{2}}}$ $\left(S_{2}\left\|S_{1}\right\| A C K\left\|R_{1}\right\| R_{2}\right)$ and sends $A C K$ and $v_{3}$ to $S_{I}$ as follows.

$$
S_{2} \rightarrow S_{1}: S_{2}\left\|S_{1}\right\| A C K \| v_{3}
$$

d. $S_{I}$ verifies $v_{3}$ and updates $N S L_{S_{1}}$.

As a result, $S_{1}$ shares a key $K_{S_{1} S_{2}}$ with $S_{2}$. The integrity key $I K_{S_{1} S_{2}}$ and the cipher key $C K_{S_{1} S_{2}}$ are derived from $K_{S_{1} S_{2}}$. If a sink receives a "HELLO" message from the sinks in the NSL, ignore this phase. Figure 5 shows a simplified activity diagram of this phase.
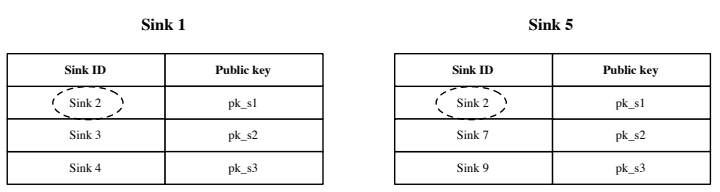

Figure 6: Sink 1 and Sink 5 find the neighbor sink Sink 2 in their Neighbor Sink Lists

3.3.3. Pre-phase 2: Initial Mobile Node Authentication. When a mobile sensor node that is only capable of symmetric key computation is joining the network for the first time, pre-phase 2 is operated as follows:

a. When a node $N$ receives $v_{0}$ from $S_{1}, N$ randomly selects $R_{1}$ and generates $u_{1}=e_{C K_{N, B S}}\left\{R_{1} \| v_{0}\right\}$, $v_{1}=M A C_{I K_{N, B S}}\left(N\left\|S_{1}\right\| u_{1}\right)$, where $C K_{N, B S} \quad$ and $I K_{N, B S}$ are the shared cipher key and integrity key, respectively, between $N$ and $B S$, and sends $u_{l}$ and $v_{l}$ to $S_{l}$ as follows.

$$
N \rightarrow S_{1}: N\left\|S_{1}\right\| u_{1} \| v_{1}
$$


b. After receiving $u_{1}$ and $v_{l}, S_{l}$ randomly selects $R_{2}$, and generates $u_{2}=e_{C K_{S_{1}, B S}}\left\{u_{1} \| R_{2}\right\} \quad$ and $v_{2}=M A C_{I K_{S_{1}}, B S}\left(S_{1}\|B S \quad\| N\left\|u_{2}\right\| v_{1}\right)$, where $C K_{S_{1}, B S}$ and $I K_{S_{1}, B S}$ are shared the cipher key and integrity key, respectively, between $S_{1}$ and $B S$. Then, $S_{l}$ sends $u_{2}, v_{l}$ and $v_{2}$ to BS as follows.

$$
S_{1} \rightarrow B S: S_{1}\|B S\| N\left\|u_{2}\right\| v_{1} \| v_{2}
$$

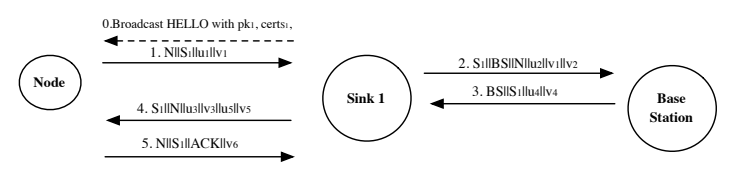

Figure 7: Pre-Phase 2: Initial Mobile Node Authentication

c. After receiving $u_{2}, v_{1}$ and $v_{2}$, BS verifies $v_{2}$ and decrypts $u_{2}$. BS then retrieves $R_{2}$ and verifies $v_{l}$. BS also retrieves $R_{1}, T S$ and $v_{0}$, and verifies $v_{0}$ checking if the $T S$ is valid. BS then generates $u_{3}=e_{C K_{N, B S}}\left\{R_{2}\right\}, \quad v_{3}=M A C_{I K_{N, B S}}\left(B S\|N\| S_{1} \| u_{3}\right)$, $u_{4}=e_{C K_{S_{1}, B S}}\left\{R_{1}\left\|u_{3}\right\| v_{3}\right\} \quad$ and $\quad v_{4}=M A C_{I K_{S_{1}, B S}}$ (BS $\left\|S_{1}\right\| N\left\|R_{2}\right\| u_{4}$ ), and sends $u_{4}$ and $v_{4}$ to $S_{1}$ as follows.

$$
B S \rightarrow S_{1}: B S\left\|S_{1}\right\| u_{4} \| v_{4}
$$

d. $\quad S_{I}$ verifies $v_{4}$ and decrypts $u_{4}$. After retrieving $R_{l}, u_{3}$ and $v_{3}, S_{l}$ derives $K_{N, S_{1}}=K D F\left(R_{1} \| R_{2}\right)$ that will be the shared session key between $S_{I}$ and N. $C K_{N, S_{1}}$ and $I K_{N, S_{1}}$ are the cipher key and integrity key, respectively, derived from $K_{N, S_{1}}$ individually. $S_{I}$ then generates $u_{5}=e_{C K_{N, S_{1}}}$ $\left\{N S L_{S_{1}}\right\}$ and $v_{5}=M A C_{I K_{N, S_{1}}}\left(S_{1}\|N\| R_{1} \| u_{5}\right) . S_{1}$ then sends $u_{3}, v_{3}, u_{5}$, and $v_{5}$ to $N$ as follows.

$$
S_{1} \rightarrow N: S_{1}\|N\| u_{3}\left\|v_{3}\right\| u_{5} \| v_{5}
$$

e. After verifying $v_{3}, N$ decrypts $u_{3}$ and retrieves $R_{2} . N$ derives $K_{N, S_{1}}$ using $R_{2} . N$ can verify $v_{5}$ and decrypt $u_{5}$. After decrypting $u_{5}, N$ obtains $N S L_{S_{1}}$ $\begin{array}{ccr}N & \text { then } & \text { generates } \\ v_{6}=M A C_{I K_{N, S_{1}}}\left(N \mid S_{1}\|A C K\| R_{2} \| R_{1}\right) & \text { and sends } A C K\end{array}$ and $v_{6}$ to $S_{1}$ as follows.

$$
N \rightarrow S_{1}: N\left\|S_{1}\right\| A C K \| v_{6}
$$

f. After $S_{1}$ verifies $v_{6}, S_{1}$ authenticates $N$.
Figure 9 shows the simplified activity diagram of this phase.

3.3.4. Reconnecting Mobile Node Authentication. When an authenticated node $N$ moves and reconnects to another sink $S_{2}, N$ and $S_{2}$ proceed as follows. Assume $N$ receives $v_{0}$ from $S_{2}$.

a. $\quad N$ randomly selects $R_{l}$, and generates $u_{1}=e_{C K_{N, S_{1}}}\left\{R_{1} \| v_{0}\right\}$ and $v_{1}=M A C_{I K_{N, S_{1}}}\left(N\left\|S_{2}\right\| t_{1} \| v_{0}\right)$. $N$ then sends $N S L_{S_{1}}, u_{1}$, and $v_{l}$ to $S_{2}$ as follows.

$$
N \rightarrow S_{2}: N\left\|S_{2}\right\| N S L_{S_{1}}\left\|u_{1}\right\| v_{1}
$$

b. $S_{2}$ verifies $N S L_{S_{1}}$ and checks whether $S_{I}$ is a neighbor sink of $S_{2}$ with the public key $p k_{S_{1}}$. If $S_{1}$ is a neighbor sink of $S_{2}, S_{2}$ generates $u_{2}=e_{C K_{S_{1}}, S_{2}}\left\{N\left\|R_{2}\right\| u_{1} \| v_{1}\right\} \quad$ and $v_{2}=M A C_{I K_{S_{1}}, S_{2}}\left(S_{2}\left\|S_{1}\right\| u_{2}\right)$, where $C K_{S_{1}, S_{2}} \quad$ and $I K_{S_{1}, S_{2}}$ are derived from $K_{S_{1}, S_{2}}$, and sends $u_{2}$ and $v_{2}$ to $S_{l}$ as follows.

$$
S_{2} \rightarrow S_{1}: S_{2}\left\|S_{1}\right\| u_{2} \| v_{2}
$$

c. After verifying $v_{2}, S_{l}$ decrypts $u_{2}$, and retrieves $N, R_{2}, u_{l}$, and $v_{l} . S_{l}$ then finds $C K_{N, S_{1}}$ and $I K_{N, S_{1}}$ to verify $v_{l}$ and decrypt $u_{l} . S_{l}$ generates $u_{3}=e_{C K_{N, S_{1}}}\left\{R_{2}\right\}, \quad v_{3}=M A C_{I K_{N, S_{1}}}\left(S_{2}\left\|R_{1}\right\| u_{3}\right)$, $u_{4}=e_{C K_{S_{1}}, S_{2}} \quad\left\{R_{1}\left\|u_{3}\right\| v_{3}\right\} \quad$ and $v_{4}=M A C_{I K_{S_{1}, S_{2}}}\left(S_{1}\left\|S_{2}\right\| N\left\|u_{4}\right\| R_{2}\right)$, and sends $u_{4}$ and $v_{4}$ to $S_{2}$.

$$
S_{1} \rightarrow S_{2}: S_{1}\left\|S_{2}\right\| u_{4} \| v_{4}
$$

d. $S_{2}$ verifies $v_{4}$ and decrypts $u_{4}$. $S_{2}$ then retrieves $R_{2}, u_{3}$ and $v_{3}$. $S_{2}$ derives a new session key $K_{N, S_{2}}=K D F\left(R_{1} \| R_{2}\right)$, and also derives $C K_{N, S_{2}}$ and $I K_{N, S_{2}}$ individually. $S_{2}$ generates $u_{5}=e_{C K_{N, S_{2}}}$ $\left\{N S L_{S_{2}}\right\} \quad$ and $\quad v_{5}=M A C_{I K_{N, S_{2}}}\left(S_{2}\|N\| u_{3}\left\|u_{5}\right\| \quad v_{3} \|\right.$ $\left.R_{1} \| R_{2}\right) . S_{2}$ then sends $u_{3}, v_{3}$, and $v_{5}$ to $N$.

$$
S_{2} \rightarrow N: S_{2}\|N\| u_{3}\left\|v_{3}\right\| v_{5}
$$

e. $N$ verifies $v_{3}$ and decrypts. $N$ generates $K_{N, S_{2}}$ and verifies $v_{5}$ using $I K_{N, S_{2}} . N$ then generates $v_{6}=M A C_{I K_{N, S_{2}}}\left(N\left\|S_{2}\right\| A C K\left\|R_{2}\right\| R_{1}\right)$ and sends $A C K$ and $v_{6}$ to $S_{2}$ as follows. 


$$
N \rightarrow S_{2}: N\left\|S_{2}\right\| A C K \| v_{6}
$$

In real environments, sensor nodes may be irregularly distributed. When $N$ requests the connection to $S_{5}, S_{5}$ may not be able to authenticate $N$ properly because $S_{5}$ may not be a neighboring sink of $S_{1}$. In this case, $S_{5}$ can authenticates $N$ via $S_{2}$ modifying step 2 as follows.

a. $S_{5}$ checks whether $S_{1}$ is a neighbor of $S_{5}$. If $S_{1}$ is not a neighbor of $S_{5}, S_{5}$ finds a common neighboring sink from $N S L_{S_{1}}$ and $N S L_{S_{5}}$. When

$S_{5}$ has a common neighbor sink $S_{2}$ with $S_{1}$ as in Figure $8, S_{5}$ randomly selects $R_{2}$ and generates $u_{2}=e_{C K_{S_{2}}, S_{5}}\left\{N\left\|R_{2}\right\| N S L_{S_{1}}\left\|u_{1}\right\| v_{1}\right\} \quad$ and $v_{2}=\operatorname{MAC}_{I K_{S_{2}}, S_{5}}\left(S_{5}\left\|S_{2}\right\| u_{2}\right) . S_{2}$ then sends $u_{2}$ and $v_{2}$ to $S_{2}$.

$$
S_{5} \rightarrow S_{2}: S_{5}\left\|S_{2}\right\| u_{2} \| v_{2}
$$

b. $S_{2}$ verifies $v_{2}$ and decrypts $u_{2} . S_{2}$ then verify $N S L_{S_{1}}$ and checks whether $S_{I}$ is a neighbor sink of $S_{2}$. If $S_{1}$ is a neighbor sink of $S_{2}, S_{2}$ generates $\quad u_{3}=e_{C_{S_{1}}, S_{2}}\left\{N\left\|R_{2}\right\| u_{1} \| v_{1}\right\} \quad$ and $v_{3}=M A C_{I_{S_{1}}, S_{2}}\left(S_{2}\left\|S_{1}\right\| u_{3}\right)$, and sends $u_{3}$ and $v_{3}$ to $S_{l}$ as follows.

$$
S_{2} \rightarrow S_{1}: S_{2}\left\|S_{1}\right\| u_{3} \| v_{3}
$$

Also, step 4 is modified such that $S_{I}$ sends the authenticating information to $S_{5}$ via $S_{2}$. We omit the details of the modified step, since the procedure is similar to the step a and $b$.

\subsection{How to Reduce Overhead in Initial Authentication}

While our protocols in $[7,8]$ could reduce overhead in re-authentication, they still have the same computation and communication overheads for initial authentication.

In order to reduce overhead of sensor nodes during initial authentication, we also proposed authentication protocol for 3G-WSN integrated networks [9]. The main idea is that the application of mobile sensor nodes such as RF4CE [19] will follow integration of multiple networks. Thus the use of the overwhelming capabilities of $3 \mathrm{G}$ networks (or $4 \mathrm{G}$ eventually) could reduce the use of communication within WSN for establishing secure channel.

\subsection{Analysis of Protocols}

Detailed analyses of protocols are shown in $[7,8]$. We do not show the detail in this paper.

\section{Related Work}

In this section, we briefly review well-known key agreement protocols designed for the sensor networks. As a commercial solution, Zigbee [3] specifies the key agreement architecture by key predistribution. In their architecture, each node preinstalls its unique keys that are shared with other entities and the network key that is shared with the entire network by the manufacturer. In order to support mobility of a node using a unique key, each node must contain as many keys as the number of nodes. Thus, most studies on authenticated key agreements attempt to increase the efficiency.

\subsection{Authenticated Key Agreement Protocols for Static Sensor Networks}

Most previous key agreement protocols were based on the symmetric key cryptosystem. Eschenauer and Gligor proposed the pairwise key agreement protocols based on the random key predistribution [5] that enabled the sharing of pairwise keys from the pre-distributed key pool. In their protocol, each node stores $m$ number of keys selected from a key pool in the initial stage. After the nodes are deployed, each node shares the key information with its neighboring nodes. When the shared keys are found, the node establishes secure links between the sinks that share the keys. After a link is established, both nodes generate a pairwise key for secure communication. However, the network establishment has a probability of failure that is increased in the case of irregular deployment of sensor nodes or unpredictable interruptions.

Zhu et al. [18] introduced the group-key-based key agreement model that minimized threats of compromised nodes. Every node has a unique key, pairwise keys with neighboring nodes, a cluster key shared with all neighboring nodes, and a global key shared with the entire network. However, they assumed that the networks are static.

Abraham and Ramanatha [1] proposed an authentication and initial shared key establishment model in hierarchical clustered networks. Ibriq and Mahgoub [11] proposed an efficient model that deployed a "partial key escrow table" for sinks. Using the key escrow table, a sink can self-generate a shared key for the attached nodes. However, all sinks have to maintain the information of every node in the table to support node mobility.

\subsection{Authenticated Key Agreement Protocols For Dynamic WSN}

4.2.1 Distributed Authentication Model. Fantacci et al. [8] proposed the distributed node authentication model that does not require the base station to act as the centralized authenticator (CA), as shown in 
Figure 2. In their model, every node shares partial authentication information of other nodes based on the secret sharing scheme [16]. A node sends an authentication request to another node; e.g., the node $\mathrm{N}_{2}$ is the authenticator and other nodes such as $\mathrm{N}_{5}$ and $\mathrm{N}_{6}$ are distributed authentication servers. The overhead on all nodes in this model is large due to their involvement in the authentication process. Since each node has to participate in the authentication procedures as an authenticator or as an authentication server, the computational and communication overhead would significantly increase as a result of frequent authentication requests. Once a node $\mathrm{N}_{1}$ is authenticated by $\mathrm{N}_{2}$, as shown in Figure 7 (a), $\mathrm{N}_{1}$ sends authentication requests to $\mathrm{N}_{7}$, as shown in Figure 7 (b). In the figure, $\mathrm{N}_{3}, \mathrm{~N}_{4}, \mathrm{~N}_{5}$, and $\mathrm{N}_{6}$ are involved in both authentication processes as authentication servers.

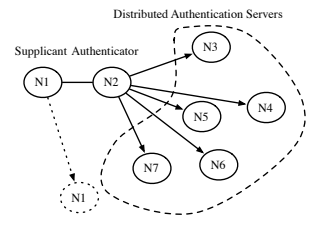

(a)



(b)
Figure 5: Fantacci's Distributed Node Authentication Model (a) Initial authentication by $N_{2}$ (b) $N_{1}$ reauthenticated by $N_{7}$

4.2.2 PKI-based Model. Although PKI brings strong and advanced security services, most studies focused on the symmetric key cryptosystem-based approach, due to the insufficient computational resources for PKI of the sensor nodes. However, many efforts that enable PKI for sensor networks such as TinyPK [17] and TinyECC [15] are often proposed.

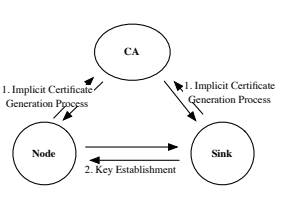

(a)

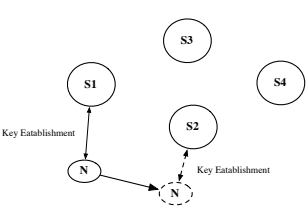

(b)
Figure 6: (a) Simplified representation of the processes of Huang's Key Agreement Model (b) Applying Huang's model in Dynamic Sensor Network

Huang et al. proposed a self-organizing algorithm by using Elliptic Curve Cryptography (ECC) [10]. Huang's model has two phases: Implicit certificate generation process and Hybrid key establishment process. Once the certificates are issued to nodes, they can self-establish the pairwise keys by exchanging the certificates with other sinks. Simplified representation of the processes is shown in Figure 9 (a). Although Huang et al. did not state that their protocol could be applicable to dynamic WSN, their protocol can support node reauthentication. After the certificate is issued to the node $N, N$ is authenticated by a sink $S_{l}$. When $N$ moves and requests the re-authentication to another sink $S_{2}, S_{2}$ can easily authenticate $N$ again as shown in Figure 9 (b).

However, their model has two critical problems: (1) all sensor nodes must contact the CA to obtain their certificates. (2) Direct contact is required between each sensor node (including mobile sensor node) and the CA, which is not considered practical for largescale networks. If an implicit certificate is preinstalled to every sink, the advantage of the protocol may be significantly reduced. The other is that every node has to be capable of ECC computation. Even though PKI-based applications for sensor networks will be available in the near future with efficient implementations, the public key-based security architecture still requires more advanced computational power and resources. A sensor node that is only capable of a lightweight cryptosystem such as AES or SHA-1 may not be able to connect to such networks.

\section{Conclusion}

While most current security protocols are designed for the static sensor networks and has several problems that applying such models in dynamic environment may occur significantly large resource drain.

In this paper, we introduced our efficient model for authenticated key agreement in dynamic WSN and showed several protocols we proposed in $[7,8]$. We showed symmetric key based protocol and hybrid protocol that combines symmetric key base model with PKI based model.

Our protocols enable the reduced authentication process for the mobile node and can be used in various application of WSN.

\section{References}

[1] Jibi Abraham and K S Ramanatha. An Efficient Protocol for Authentication and Initial Shared Key Establishment in Clustered Wireless Sensor Networks. Proceeding of Third IFIP/IEEE International Conference on Wireless and Optical Communications Networks, 2006.

[2] Ian F. Akyildiz and Ismail H. Kasimoglu. Wireless sensor and actor networks: research challenges. Ad Hoc Networks, 2(4):351 - 367, 2004.

[3] William C Craig. Zigbee:Wireless Control That Simply Works. Zigbee Alliance, 2005.

[4] Jeremy Elson and Deborah Estrin. Random, Ephemeral Transaction Identifiers in Dynamic Sensor Networks. 21st International Conference on Distributed Computing Systems, :pp. 0459, 2001. 
[5] L. Eschenauer and V.D. Gligor. A key management scheme for distributed sensor networks". in Proceedings of the 9th ACM conference on Computer and Communications Security (CCS). Washington. DC. USA, :41-47, 2002.

[6] Gill, K. and Shuang-Hua Yang and Fang Yao and Xin Lu. A Zigbee-based home automation system. IEEE Transactions on Consumer Electronics, 55(2):422430, 2009.

[7] Kyusuk Han and Kwangjo Kim and Taeshik Shon. Untraceable Mobile Node Authentication in WSN. Sensors, 10(5):4410-4429, 2010.

[8] Kyusuk Han and Taeshik Shon and Kwangjo Kim. Efficient Mobile Sensor Authentication In Smart Home and WPAN. IEEE Trans. on Consumer Electronics, 56(2):591-596, 2010.

[9] Kyusuk Han, Kwangjo Kim, Wook Choi, Hyohyun Choi, Jungtaek Seo, Taeshik Shon, Efficient Authenticated Key Agreement Protocols for Dynamic Wireless Sensor Networks, Ad Hoc \& Sensor Wireless Networks, Volume 12 , Number 3 , Mar 2012, pp , ISSN: 1551-9899

[10] Qiang Huang and Johnas Cukier and Hisashi Kobayashi and Bede Liu and Jinyun Zhang. Fast authenticated key establishment protocols for selforganizing sensor networks. WSNA '03: Proceedings of the 2nd ACM international conference on Wireless sensor networks and applications, 2003.

[11] J. Ibriq and I. Mahgoub. A Hierarchical Key Establishment Scheme for Wireless Sensor Networks. Proceedings of 21st International Conference on Advanced Networking and applications (AINA'07), :210-219, 2007.

[12] C. Karlof and D. Wagner. Secure routing in wireless sensor networks. In Proc. of SNPA'03, Anchorage, Alaska, :113-127, 2003.

[13] Chris Karlof and Naveen Sastry and David Wagner. TinySec: a link layer security architecture for wireless sensor networks. SenSys '04: Proceedings of the 2nd international conference on Embedded networked sensor systems, 2004.

[14] HangRok Lee and YongJe Choi and HoWon Kim. Implementation of TinyHash based on Hash Algorithm for Sensor Network. Proceedings of World Academy of Science, Engineering and Technology, 10:135-139, 2005.

[15] An Liu and Peng Ning. TinyECC: A Configurable Library for Elliptic Curve Cryptography in Wireless Sensor Networks. 2008 International Conference on Information Processing in Sensor Networks, 2008.

[16] Adi Shamir. How to share a secret. Communications of the ACM, 22(11):612--613, 1979.

[17] Ronald Watro and Derrick Kong and Sue-fen Cuti and Charles Gardiner and Charles Lynn and Peter Kruus. TinyPK: Securing sensor networks with public key technology. Proceedings of the 2nd ACM workshop on Security of ad hoc and sensor networks, :59-64, 2004.

[18] Zhu, Sencun and Setia, Sanjeev and Jajodia, Sushil. LEAP+: Efficient security mechanisms for largescale distributed sensor networks. ACM Trans. Sen.
Netw., 2(4):500--528, 2006.

[19] ZigBee alliance. RF4CE Standard Specification Release 1.0. 2009. 